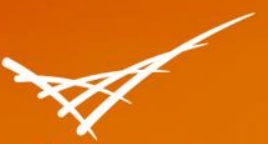

Pacific Northwest

\title{
U-10Mo Sample Preparation and Examination using Optical and Scanning Electron Microscopy
}

\section{March 2016}

R Prabhakaran

VV Joshi

MA Rhodes

AL Schemer-Kohrn

A Guzman

CA Lavender 


\title{
DISCLAIMER
}

This report was prepared as an account of work sponsored by an agency of the United States Government. Neither the United States Government nor any agency thereof, nor Battelle Memorial Institute, nor any of their employees, makes any warranty, express or implied, or assumes any legal liability or responsibility for the accuracy, completeness, or usefulness of any information, apparatus, product, or process disclosed, or represents that its use would not infringe privately owned rights. Reference herein to any specific commercial product, process, or service by trade name, trademark, manufacturer, or otherwise does not necessarily constitute or imply its endorsement, recommendation, or favoring by the United States Government or any agency thereof, or Battelle Memorial Institute. The views and opinions of authors expressed herein do not necessarily state or reflect those of the United States Government or any agency thereof.

\author{
PACIFIC NORTHWEST NATIONAL LABORATORY \\ operated by \\ BATTELLE \\ for the \\ UNITED STATES DEPARTMENT OF ENERGY \\ under Contract DE-AC05-76RL01830
}

Printed in the United States of America
Available to DOE and DOE contractors from the Office of Scientific and Technical Information, P.O. Box 62, Oak Ridge, TN 37831-0062; ph: (865) 576-8401 fax: $(865) 576-5728$
email: reports $a$ adonis.osti.gov
Available to the public from the National Technical Information Service 5301 Shawnee Rd., Alexandria, VA 22312 ph: (800) 553-NTIS (6847) email: orders@ntis.gov <http://www.ntis.gov/about/form.aspx> Online ordering: http://www.ntis.gov




\title{
U-10Mo Sample Preparation and Examination using Optical and Scanning Electron Microscopy
}

\author{
R Prabhakaran \\ VV Joshi \\ MA Rhodes \\ AL Schemer-Kohrn \\ A Guzman \\ CA Lavender
}

March 2016

Prepared for

the U.S. Department of Energy

under Contract DE-AC05-76RL01830

Pacific Northwest National Laboratory

Richland, Washington 99352 



\section{Purpose/Scope}

The purpose of this document is to provide guidelines to prepare specimens of uranium alloyed with 10 weight percent molybdenum (U-10Mo) for optical metallography and scanning electron microscopy. This document also provides instructions to set up an optical microscope and a scanning electron microscope to analyze U-10Mo specimens and to obtain the required information.

\section{Prerequisites and Precautions}

Staff members who perform work in accordance with this procedure must be qualified. Assigned staff shall read and comply with the applicable work control procedures and other applicable operating procedures.

Instructions for operating the equipment can be found in the manufacturer's instruction manual. Do not operate the sectioning saw in the presence of flammable liquids, gases, or dust.

Working with radioactive materials/sources can cause doses of radiation to one's skin and extremities. Care should be taken to identify these materials. Avoid direct handling and use remote handling tools, if possible.

Samples can become scratched or damaged very easily. Samples must be kept clean and stored properly for examination. Confirm that all specimens have been properly cleaned before metallography.

Good housekeeping practices shall be followed during all operations in the fume hood, glove box, and bench top. 



\section{Acronyms and Abbreviations}

BSE

DIC

DIW

EBSD

EDS

LABE

LEI

LM

Mo

PSA

SDD

SEM

$\mathrm{U}-10 \mathrm{Mo}$

WD

WDS backscattered electron

differential interference contrast

deionized water

electron backscatter diffraction

energy-dispersive x-ray spectroscopy

low-angle backscattered electron

lower electron image

Low Mag mode

molybdenum

pressure sensitive adhesive

silicon drift detector

scanning electron microscopy

uranium alloyed with 10 weight percent molybdenum

working distance

wavelength-dispersive $\mathrm{x}$-ray spectroscopy detector 



\section{Contents}

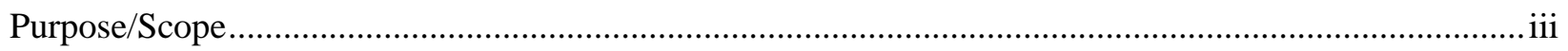

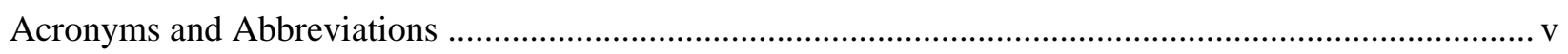

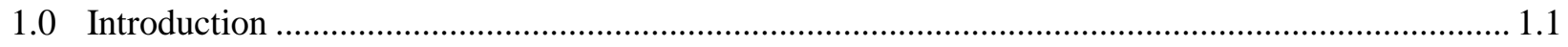

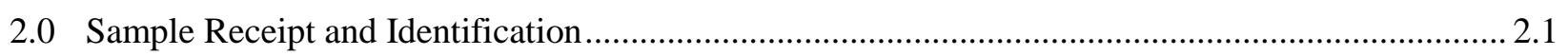

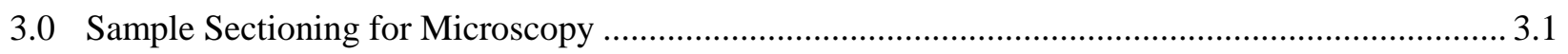

4.0 Sample Mounting for Microscopy - Bare U-Mo.......................................................................... 4.1

5.0 Sample Mounting and Slicing for Microscopy - Plated U-Mo ................................................... 5.1

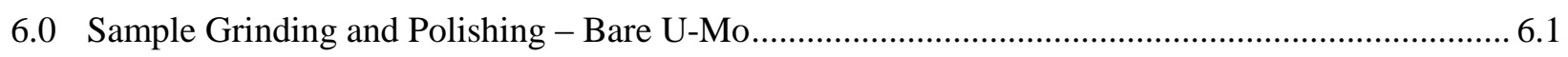

7.0 Sample Grinding and Polishing - Plated U-Mo .................................................................... 7.1

8.0 Sample Final Polishing - Bare U-Mo................................................................................. 8.1

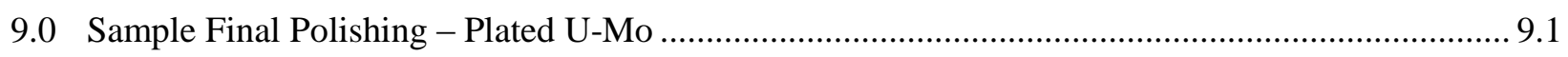

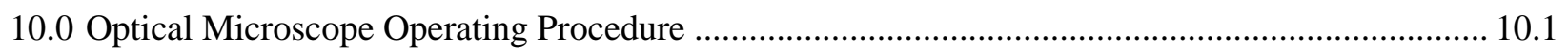

11.0 Scanning Electron Microscope Operating Procedure ............................................................... 11.1

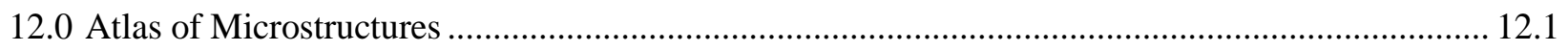




\section{Figures}

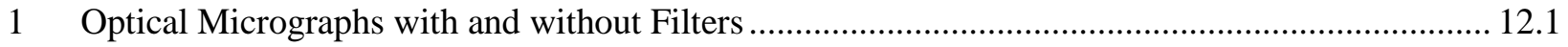

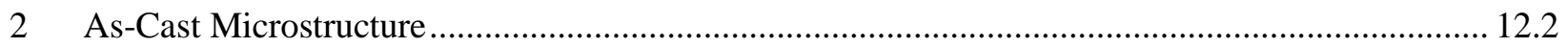

3 As-Cast Microstructure: Effect of Electron Voltage on Microstructure ...................................... 12.3

4 U-10Mo Microstructure: Effect of Polarized Light; BSE-SEM vs. Secondary Electron Images.... 12.4

$5 \quad$ U-10Mo Microstructure: As-Cast and Rolled Microstructure ..................................................... 12.5

6 U-10Mo Microstructure: As-Cast, Homogenized, and Rolled Microstructure ............................... 12.6

$7 \quad$ U-10Mo Microstructure: Eutectoid Structure ............................................................................ 12.7

\section{Tables}

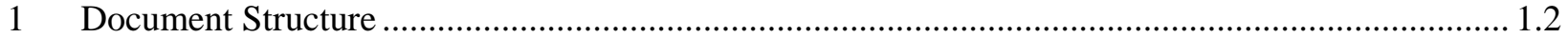




\subsection{Introduction}

Pacific Northwest National Laboratory has been investigating manufacturing processes for a plate-type fuel made from a uranium alloy with 10 weight percent molybdenum (U-10Mo). This work supports the Reactor Conversion Program (RC) of the U.S. Department of Energy National Nuclear Security Administration's Office of Material Management and Minimization. Use of metallic fuel is required to meet the objectives of RC for the fleet of high-performance research reactors in the United States. The metallic fuel selected to replace the current fuels is the low-enriched uranium 10-weight percent molybdenum alloy (LEU-Mo) in a thin sheet or foil form encapsulated in AA6061 aluminum alloy. The Fuel Fabrication Capability Pillar of RC has undertaken a series of tasks in order to meet performance and schedule requirements, and a series of projects have been undertaken that increase the understanding of the impact of processing conditions on the final fuel microstructure.

This report describes the process to prepare U-10Mo metallographic samples. Metallography is the study of metals by using optical and electron microscopes. Structures that are coarse enough to be discernible to the naked eye or under low magnifications are termed macrostructures, whereas those that require high magnification to be visible are termed microstructures. Microscopes are required for the examination of a metal's or alloy's microstructure. Optical metallography and scanning electron microscopy play a vital role in studying a material's microstructure.

By examining and quantifying a material's microstructure, its performance can be better understood. Thus, metallography can be used in all stages during the lifetime of a component: from the initial materials development to the inspection, production, manufacturing process control, and even failure analysis if needed. The principles of metallography play a vital role in attaining product reliability.

The basic steps for performing proper microstructural analysis include sample receipt, identification, and documentation; sample sectioning; sample mounting; sample grinding and polishing; sample final polishing; sample etching; and microscopic analysis.

Sampling, the first step of metallographic analysis, is critical to the success of any subsequent study. Care must be taken to make sure that the specimen selected for analysis is representative of the material being evaluated. The second, equally significant, step is to properly prepare the metallographic specimen, and here there is no unique way to achieve the desired results. Metallography has been traditionally described as both a science and an art, because experience and intuition are equally significant to expose the true structure of the material without causing substantial change or damage.

The purpose of this document is to provide guidelines to prepare U-10Mo specimens for optical metallography and scanning electron microscopy. This document also provides instructions to set up an optical microscope and scanning electron microscope to analyze U-10Mo specimens, and to obtain the required information. This document can be used to help prepare and analyze both bare U-Mo and plated U-Mo samples, by referring to the appropriate sections, as shown in Table 1 below. 
Table 1. Document Structure

\begin{tabular}{c|ll}
\hline $\begin{array}{c}\text { Type of } \\
\text { Sample }\end{array}$ & & \multicolumn{1}{c}{ Applicable Sections } \\
\hline \multirow{4}{*}{ Bare U-Mo } & Section 2 & Sample Receipt and Identification \\
& Section 3 & Sample Sectioning for Microscopy \\
& Section 4 & Sample Mounting for Microscopy - Bare U-Mo \\
& Section 6 & Sample Grinding and Polishing - Bare U-Mo \\
& Section 10 & Sample Final Polishing - Bare U-Mo \\
& Section 11 & Scanning Electron Microscope Operating Procedure \\
& Section 12 & Atlas of Microstructures \\
\hline & Section 2 & Sample Receipt and Identification \\
& Section 3 & Sample Sectioning for Microscopy \\
& & Sample Mounting and Slicing for Microscopy - Plated U- \\
& Section 5 & Mo \\
& Section 7 & Sample Grinding and Polishing - Plated U-Mo \\
& Section 9 & Sample Final Polishing - Plated U-Mo \\
& Section 10 & Optical Microscope Operating Procedure \\
& Section 11 & Scanning Electron Microscope Operating Procedure \\
& Section 12 & Atlas of Microstructures \\
\hline
\end{tabular}




\subsection{Sample Receipt and Identification}

Upon receipt of a sample (bare U-Mo or plated U-Mo), an entry shall be made in the Sample Log. The Sample Log should contain the sample identification number, sample description, and other relevant information to get positive sample identity and traceability. The Sample Log should mention the type of preparation required, as determined by the cognizant engineer. The sample and/or sample container shall be marked with a sample identification number and be tagged. The location of the sample shall be recorded in the Sample Log. 



\subsection{Sample Sectioning for Microscopy}

As-received samples (bare U-Mo and plated U-Mo) usually require sectioning to obtain pieces of appropriate size for sample preparation. Proper sectioning is required to minimize damage, which may alter the material's microstructure and can produce false metallographic characterization. Proper cutting requires the correct selection of abrasive type, bonding, and size, as well as proper cutting speed, load, and coolant. Make sure to choose appropriate sectioning/cutting equipment based on sample dimensions (primarily the thickness).

This section of the procedure applies to the shearing, cutting, sectioning, or machining of radioactive samples in the fume hood or glove box using a low-speed saw, band saw, or bench shear. A contamination area or high contamination area may be created during the sectioning of the various materials. Sectioning operations are strictly limited to the fume hood or glove box.

\subsection{Equipment/Supplies}

- bench shear

- Buehler IsoMet low-speed saw

- UKAM Smart Cut 6001 GP sectioning saw (for large pieces)

- water

- terry towel (or similar absorbent material)

- lubricating solution

\subsection{Preparation of the Cutting Equipment}

3.2.1 Verify that the cutting equipment is working properly. Refer to its manual for instructions related to the operation of the equipment.

3.2.2 In the fume hood, the equipment should be placed in such a way that the radioactive material is at least 6 inches from the front sill of the fume hood and that the airflow baffles at the rear of the fume hood are not blocked.

3.2.3 Prepare the fume hood or glove box by covering the area around the equipment with absorbent material to control the spread of contamination. For example, a terry towels or similar absorbent material may be placed around the equipment.

3.2.4 If appropriate, provide lubrication for the cutting blade or surface.

3.2.4.1 For the saw blade, either add water to the lubrication pan or place a wet towel into the pan to lubricate the blade and collect the cutting residue. A paper towel can also be folded and inserted, so that it can wipe the blade and minimize spraying of lubricant from the sample during sawing. 


\subsection{Retrieving Materials for Sectioning}

3.3.1 Unpack the samples and transfer to the fume hood or glove box. If contamination is suspected or levels of contamination are unknown, the innermost, or sample, package of concern may be opened in the fume hood, glove box, or other contamination area. Make sure to follow appropriate procedures while working with radioactive materials.

\subsection{Sectioning}

3.4.1 Specimens shall be sectioned as requested to expose the appropriate surfaces for the metallographic analysis or for other testing requirements. The orientation of the cut section with respect to the original sample shall be recorded.

3.4.2 Position the sample in the cutting fixture so that the desired length and surface of interest will be exposed after the specimen is cut and polished. Clamp the sample to be sectioned in the sample chuck.

3.4.3 Before operating the equipment to section the material, make sure that the blade can remain lubricated. Make sure that the lubricant is contained by adjusting the blade speed to prevent splashing and spraying from the blade. A shield can be used to control the spread of contamination. The approach, "as low as reasonably achievable possible" should apply to minimize the personal dose.

3.4.4 Turn on the cutting equipment and gently lower the specimen onto the rotating blade (or, as applicable, gently lower the blade onto the specimen).

3.4.5 When operating the saw, when the blade is close to cutting through the specimen, decrease the speed of the blade, hold on to the cut section, and lift the fulcrum arm to prevent a back-slash cut to the specimen.

3.4.6 When operating the bench shear, make sure to use appropriate gloves and keep your fingers away from the cutting blade.

3.4.7 Multiple cuts may be required to section the sample.

\subsection{Job Completion and Cleanup}

3.5.1 Once sectioning is complete, remove the sample from the chuck. Collect all sectioned pieces. Clean the work area to reduce/remove contamination and arrange for a survey. Based on the survey, determine whether the contamination is as low as reasonably achievable or further cleaning is required. After cleaning, store the sample in a clean, clearly labeled bag/container. Transfer, if needed. Record the ID and location of the cut pieces in the Sample Log.

3.5.2 Place any unused pieces (in a labeled bag) in a shielded area of the fume hood, glove box, or other shielded location. Record the sample ID and location of these pieces in the Sample Log. 
3.5.3 Place any waste material in a waste container in the fume hood or glove box. If the waste material has a high dose rate, segregate it from the other waste material and shield it until the material can be removed for disposal.

3.5.4 Clean the equipment, chuck, and tools by washing and wiping the contaminated areas.

3.5.5 When using the saw, remove excess liquids from the saw pan by blotting up the liquids with an absorbent material. If a damp towel was used to assist in the lubrication, dispose it using an absorbent material in a radioactive waste bag clearly marked with a description of contents, source of material, and contamination level.

3.5.5.1 Rinse the pan with water and blot up the rinse water.

3.5.5.2 Remove the pan and place it in a plastic bag.

3.5.6 Remove the absorbent material placed around the equipment and dispose it in the fume hood or glove box waste container. Wipe the exterior surface of the equipment and surrounding area with a damp towel.

3.5.7 The cleaned equipment may be stored by moving it to a back corner of the fume hood or glove box. 



\subsection{Sample Mounting for Microscopy - Bare U-Mo}

Bare U-Mo samples are typically mounted in an epoxy resin for grinding and polishing to expose the sample surface for observation (optical or/and scanning electron microscopy). One of the main objectives of a mounting operation is to protect the sample edge and to maintain the integrity of a material's surface features. Sample mounting should be performed in a fume hood and/or benchtop area, according to the operational procedure.

\subsection{Equipment/Supplies}

- Buehler EpoThin 2 resin (Product \# 203440128)

- Buehler EpoThin 2 hardener (\# 203442064)

- Buehler SamplKup 1.25" mounts (\# 208180)

- Buehler release agent (\# 208185016)

- Buehler SamplKlip stainless support clip (\# 204000100)

- Buehler SamplKlip I plastic support clip (\# 204100100)

- Buehler disposable paper cups (\# 208177100)

- Buehler disposable stirring sticks (\# 208175)

- bell jar vacuum chamber

- electronic engraver

- ethanol

- compressed air

- Marker (eg. Sharpie)

\subsection{Mounting Bare U-Mo Samples}

4.2.1 Mix the mounting resin and hardener according to the manufacturer's recommendation using a stirring stick in a disposable paper cup.

4.2.2 Clean the 1.25" sample mount cup using ethanol and dry it with compressed air. Apply the release agent to the sample mount cup (inner surface).

4.2.3 Write the associated sample identification number on the mounting cup with a marker or electronic engraver.

4.2.4 Clean the sample using ethanol and dry it with compressed air.

4.2.5 Place the sample in the mount with the surface (longitudinal, transverse, etc.) that requires examination facing the bottom of the mounting cup. If needed, use an appropriate support clip to hold the sample upright.

4.2.6 Dispense the mixed epoxy system into the mounting cup containing the sample with a mount thickness of about $0.75 "$. 
4.2.7 A vacuum chamber can be used to remove the air bubbles around the sample inside the mount.

4.2.8 Allow the epoxy system to cure as directed (minimum 9 hours at room temperature before removing from the mounting cup; suggested minimum 48 hours prior to grinding).

4.2.9 Once the epoxy curing process is complete, write the sample identification number on the exposed hardened face of the epoxy with a marker or electronic engraver, then remove the mounted sample from the mounting cup.

4.2.10 Use an electronic engraver to inscribe the sample identification number on the side of the mounted sample. 


\subsection{Sample Mounting and Slicing for Microscopy - Plated U-Mo}

Plated U-Mo samples are typically mounted in an epoxy resin for grinding and polishing to expose the sample surface for observation (optical or/and scanning electron microscopy). One of the main objectives of a mounting operation is to protect the sample edge and to maintain the integrity of a material's surface features (e.g., plating). Sample mount slicing is performed to remove portions of the mounted sample surface that have cutting damage (such as shear damage). Sample mounting and slicing should be performed in a fume hood and/or benchtop area, according to the operational procedure.

\subsection{Equipment/Supplies}

- Buehler EpoThin 2 resin (\# 203440128)

- Buehler EpoThin 2 hardener (\# 203442064)

- Buehler SamplKup 1.25" mounts (\# 208180)

- Buehler release agent (\# 208185016)

- Buehler SamplKlip stainless support clip (\# 204000100)

- Buehler SamplKlip I plastic support clip (\# 204100100)

- Buehler disposable paper cups (\# 208177100)

- Buehler disposable stirring sticks (\# 208175)

- Buehler IsoMet low-speed saw

- Buehler mount chuck (\# 11-1189)

- Buehler IsoCut fluid (\# 11-1193-032) or appropriate non-water-based cutting fluid

- bell jar vacuum chamber

- electronic engraver

- microscope glass slides

- ethanol

- compressed air

- marker (e.g. Sharpie)

\subsection{Mounting Plated U-Mo Samples}

5.2.1 Mix the mounting resin and hardener according to the manufacturer's recommendation using a stirring stick in a disposable paper cup.

5.2.2 Clean the 1.25" sample mount cup with ethanol and dry it with compressed air. Apply the release agent to the sample mount cup (inner surface).

5.2.3 Write the associated sample identification number on the mounting cup with a marker or electronic engraver. 
5.2.4 Pre-cut glass slides into smaller sections. These will be used for edge retention.

5.2.5 Clean the plated U-Mo sample using ethanol and dry it with compressed air.

5.2.6 Place a glass section on each of the two side faces of the sample. Use a plastic spring clip on each end to sandwich the sample between the two glass sections.

5.2.7 Place the sample assembly (sample with glass sections and clips) inside the sample mount cup with the surface that requires examination facing the bottom of the mounting cup.

5.2.8 Dispense the mixed epoxy system into the mounting cup containing the sample assembly with a mount thickness of about $0.75 "$.

5.2.9 A vacuum chamber can be used to remove the air bubbles that surround the sample assembly inside the mounting cup.

5.2.10 Allow the epoxy system to cure as directed (minimum 9 hours at room temperature before removing from the mounting cup; suggested minimum 48 hours prior to grinding).

5.2.11 Once the epoxy curing process is complete, with a marker or electronic engraver the sample identification number on the exposed face of the hardened epoxy, then remove the mounted sample from the mounting cup.

5.2.12 Use an electronic engraver to inscribe the sample identification number on the side of the mounted sample.

\subsection{Slicing Mounted Plated U-Mo Samples}

5.3.1 Install the mounted sample into the mount chuck and secure into the Buehler IsoMet low-speed saw.

5.3.2 Use Buehler IsoCut fluid or appropriate non-water-based cutting fluid as the lubricant.

5.3.3 Slice off approximately $0.125 "(3 \mathrm{~mm})$ from the mounted sample to remove portions that have cutting damage (such as shear damage). The low-speed saw is a precision sectioning saw designed for cutting various types of materials with minimal deformation.

5.3.4 Once the slicing process is complete, inspect the face of the mounted sample for any voids near interfaces and clips. If voids are present, clean and pour a small amount of epoxy mixture (resin and hardener) onto the epoxy mount face and repeat the vacuum impregnation process (steps 5.2.9 and 5.2.10)

5.3.5 Allow the epoxy system to cure as directed (minimum 24-48 hours). 


\subsection{Sample Grinding and Polishing - Bare U-Mo}

Mounted bare U-Mo samples require grinding and polishing to expose the sample surface for observation (optical or/and scanning electron microscopy). Sample grinding and polishing should be performed in a radiological fume hood, by hand (manually) or using a semiautomatic grinding and polishing system. This report will focus on grinding and polishing mounted bare U-Mo samples by hand.

\subsection{Equipment/Supplies}

6.1.1 Silicon carbide grinding papers

- LECO 8" premium grade, plain-back 180 grit (\# 810-221-PRM)

- LECO 8" premium grade, plain-back 240 grit (\# 810-223-PRM)

- LECO 8" premium grade, plain-back 400 grit (\# 810-227-PRM)

- LECO 8" premium grade, plain-back 600 grit (\# 810-229-PRM)

- LECO 8" premium grade, plain-back 1200 grit (\# 810-036-100)

6.1.2 Diamond compounds/suspensions

- Buehler MetaDi Supreme Poly Diamond suspension, 9 micron (\# 40-6633)

- Buehler MetaDi Supreme Poly Diamond suspension, 3 micron (\# 40-6631)

6.1.3 Polishing cloth

- Buehler TriDent 8", pressure sensitive adhesive (PSA) Backing (\# 40-7518)

6.1.4 Cleaning solutions

- deionized water (DIW)

- ethanol

6.1.5 Others

- glass plate (for flat surface - while hand grinding and polishing)

- ultrasonic sample cleaner (example: Branson Model 2800)

- glass beaker

\subsection{Preparation of the Work Space for Mounted Bare U-Mo Sample Grinding and Polishing (by Hand)}

6.2.1 Verify the inward flow of air to the fume hood.

6.2.2 Prepare the fume hood by covering the work area with an absorbent material.

6.2.3 Prepare a waste receptacle. 
6.2.4 Make sure that the radioactive material is at least 6 inches from the front sill of the fume hood and that the air flow baffles at the rear of the fume hood are not blocked.

\subsection{Mounted Bare U-Mo Sample Grinding (by Hand)}

6.3.1 A glass plate will be used as a grinding base to help maintain sample flatness.

6.3.2 Take the sample mounted in epoxy and grind the back side using a 180 grit paper and DIW as a lubricant to flatten the back surface and to smooth the edges of the epoxy mount. An uneven back surface can give rise to issues while performing microscopy or microhardness testing. A small benchtop lathe may be used to trim the back side to a uniform surface.

6.3.3 The next step is to grind the sample face by following the outline (shown below) using DIW as a lubricant. Since grinding/polishing pressure is dependent upon the applied force and the area of the specimen and mounting material, the operator may have to adjust time appropriately.

- Use 240 grit for 1 minute.

- Use 400 grit for 1 minute.

- Use 600 grit for 2 minutes.

- Use 1200 grit for 2 minutes.

6.3.4 Clean the sample properly after each stage of grinding using DIW and ethanol to avoid cross-contamination of abrasives. Ultrasonic cleaning is strongly recommended.

6.3.5 Discard the used grinding paper in the waste receptacle. Clean the work area to prevent cross-contamination of abrasives.

6.3.6 Rotate the sample $90^{\circ}$ between successive grit sizes. If necessary, the abrasive paper can be replaced with a newer piece to increase cutting rates.

6.3.7 The surface of the sample should be flat and evenly ground/polished before proceeding to a smaller grit of paper. Repeat as necessary.

\subsection{Mounted Bare U-Mo Sample Polishing (by Hand)}

6.4.1 A glass plate will be used as a polishing base to help maintain sample flatness.

6.4.2 Take one TriDent polishing cloth and apply 9-micron diamond suspension to coat the surface of the cloth. Polish sample for 1 minute and clean with DIW and ethanol.

6.4.3 Store the polishing cloth in a properly labeled storage bag for future use. Clean the work area to prevent cross-contamination of abrasives.

6.4.4 Take another TriDent polishing cloth and apply 3-micron diamond suspension to coat the surface of the cloth. Polish sample for 1 minute and clean with DIW and ethanol. 
6.4.5 Store the polishing cloth in a properly labeled storage bag for future use. Make sure to use different bags for cloths employed for different diamond suspensions to prevent cross-contamination of abrasives.

\subsection{Cleanup of the Workspace after Mounted Bare U-Mo Sample Grinding and Polishing (by Hand)}

6.5.1 After completing the grinding and polishing process, clean the glass plate.

6.5.2 Remove the absorbent material covering the work area and place it in the waste receptacle.

6.5.3 Use terry towels to clean the work area.

6.5.4 Arrange for a radiological survey. Based on the survey, determine whether the contamination is as low as reasonably achievable or further cleaning is required. 



\subsection{Sample Grinding and Polishing - Plated U-Mo}

Plated U-Mo samples require grinding and polishing to expose the sample surface for observation (optical or/and scanning electron microscopy). Sample grinding and polishing should be performed in a radiological fume hood, by hand (manually) or using a semiautomatic grinding and polishing system. Plated U-Mo samples should be prepared using non-water-based lubricants and polishing suspensions. This report will focus on preparing plated U-Mo samples by hand.

\subsection{Equipment/Supplies}

7.1.1 Silicon carbide grinding papers

- LECO 8" premium grade, plain-back 180 grit (\# 810-221-PRM)

- LECO 8" premium grade, plain-back 240 grit (\# 810-223-PRM)

- LECO 8" premium grade, plain-back 400 grit (\# 810-227-PRM)

- LECO 8" premium grade, plain-back 600 grit (\# 810-229-PRM)

- LECO 8" premium grade, plain-back 1200 grit (\# 810-036-100)

7.1.2 Diamond compounds/suspensions

- Buehler MetaDi monocrystalline diamond oil-based suspension, 9 micron (\# 40-6543)

- Buehler MetaDi monocrystalline diamond oil-based suspension, 3 micron (\# 40-6541)

- Alternate: Allied diamond compound, 9 micron (\# 90-21030-S)

- Alternate: Allied diamond compound, 3 micron (\# 90-21020-S)

7.1.3 Polishing cloth

- Buehler TriDent 8", PSA backing (\# 40-7518)

7.1.4 Lubricant

- Wendt Dunnington (3M) lapping oil OS114, 1 gal (\# 185029)

7.1.5 Others

- glass plate (for flat surface - while hand grinding and polishing)

- ultrasonic sample cleaner (example: Branson Model 2800)

- ethanol

- glass beaker

\subsection{Preparation of the Workspace for Plated U-Mo Sample Grinding and Polishing (by Hand)}

7.2.1 Verify the inward flow of air to the fume hood. 
7.2.2 Prepare the fume hood by covering the work area with an absorbent material.

7.2.3 Prepare a waste receptacle.

7.2.4 Make sure that the radioactive material is at least 6 inches from the front sill of the fume hood and that the air flow baffles at the rear of the fume hood are not blocked.

\subsection{Plated U-Mo Sample Grinding (by Hand)}

7.3.1 A glass plate will be used as a grinding base to help maintain sample flatness.

7.3.2 Take the sample mounted in epoxy and grind the back side using a 180-grit paper and lapping oil as a lubricant to flatten the back surface and to smooth the edges of the epoxy mount. An uneven back surface can give rise to issues while performing microscopy or microhardness testing.

7.3.3 The next step is to grind the sample face by following the outline (shown below) by using lapping oil as a lubricant. Do not use water as a lubricant for plated U-Mo samples. Since grinding/polishing pressure is dependent upon the applied force and the area of the specimen and mounting material, the operator may have to adjust time appropriately.

- Use 240 grit for 1 minute.

- Use 400 grit for 1 minute.

- Use 600 grit for 2 minutes.

- Use 1200 grit for 2 minutes.

7.3.4 Clean the sample properly after each stage of grinding using ethanol to avoid crosscontamination of abrasives. Do not use water for cleaning the plated U-Mo samples. Ultrasonic cleaning is strongly recommended.

7.3.5 Discard the used grinding paper in the waste receptacle. Clean the work area to prevent cross-contamination of abrasives.

7.3.6 Rotate the sample $90^{\circ}$ between successive grit sizes. If necessary, the abrasive paper can be replaced with a newer piece to increase cutting rates.

7.3.7 The surface of the sample should be flat and evenly ground/polished before proceeding to a smaller grit of paper. Repeat as necessary.

\subsection{Plated U-Mo Sample Polishing (by Hand)}

7.4.1 A glass plate will be used as a polishing base to help maintain sample flatness.

7.4.2 Take one TriDent polishing cloth and apply 9-micron oil-based diamond suspension, or 9-micron compound with lapping oil, to coat the surface of the cloth. Polish sample for 1 minute and clean with ethanol. Do not use water for cleaning the sample. 
7.4.3 Store the polishing cloth in a properly labeled storage bag for future use. Clean the work area to prevent cross-contamination of abrasives.

7.4.4 Take another TriDent polishing cloth and apply 3-micron oil-based diamond suspension, or 3-micron compound with lapping oil, to coat the surface of the cloth. Polish sample for 1 minute and clean with ethanol. Do not use water for cleaning the sample.

7.4.5 Store the polishing cloth in a properly labeled storage bag for future use. Make sure to use different bags for cloths employed for different diamond suspensions to prevent cross-contamination of abrasives.

\subsection{Cleanup of the Workspace after Plated U-Mo Sample Grinding and Polishing (by Hand)}

7.5.1 After completing the grinding and polishing process, clean the glass plate.

7.5.2 Remove the absorbent material covering the work area and place it in the waste receptacle.

7.5.3 Use terry towels to clean the work area.

7.5.4 Arrange for a radiological survey. Based on the survey, determine whether the contamination is as low as reasonably achievable or further cleaning is required. 



\subsection{Sample Final Polishing - Bare U-Mo}

The purpose of final polishing on bare U-Mo mounted samples is to remove the surface damage only. It should not be used to remove any damage during sectioning and grinding operations.

\subsection{Equipment}

- Buehler VibroMet 2 vibratory polishers

\subsection{Supplies}

8.2.1 Polishing cloths

- Buehler nylon 12", PSA backing (\# 40-7072)

- Wendt Dunnington (3M) 12" Final B adhesive backing (\# 150237)

8.2.2 Polishing solutions/paste

- Buehler MetaDi II monocrystalline-diamond medium-concentration paste, 1 micron (\# 40-6243)

- Wendt Dunnington (3M) lapping oil OS114 1 gal (\# 185029)

- LECO colloidal silica polishing fluid, 0.08 micron (\# 812-121-300)

- $1 \%$ acetic acid and DIW mixture

8.2.3 Cleaning solutions

- DIW

- ethanol

- soapy water

8.2.4 Others

- Buehler weighted specimen holder 1.25" (\# 67-1527)

- Buehler loading plate/fixture (\# 67-1540)

- ultrasonic sample cleaner (example: Branson Model 2800)

- soft cloth

- terry towels

- glass plate

- glass beaker

\subsection{Prepare Vibratory Polishers for Bare U-Mo Samples}

8.3.1 Prepare the first vibratory polisher by properly applying nylon cloth without any wrinkles. Care must be taken to ensure a flat surface without any air bubbles. 
8.3.2 Apply 1-micron diamond paste onto the cloth and then fill the basin as appropriate with the lapping oil.

8.3.3 Turn on the first vibratory polisher and let it run for 15 minutes before inserting sample(s).

8.3.4 Prepare the second vibratory polisher by properly applying Final B polishing cloth. Care must be taken to ensure a flat surface without any air bubbles. Wet the cloth with water and clean off any loose fibers or debris.

8.3.5 Fill the basin of second vibratory polisher with 50/50 colloidal silica and DIW appropriately.

8.3.6 Turn on the second vibratory polisher and let it run for 15 minutes before inserting sample(s).

\subsection{Final Polishing Bare U-Mo Samples using 1 Micron Vibratory Polisher}

8.4.1 Load the weighted holder as directed in the instruction manual using the sample loading plate.

8.4.2 Once loaded, place the weighted holder with sample(s) into the first vibratory polisher ( 1 micron) and carry out polishing for 24 hours.

8.4.3 When the polishing (1 micron) is complete, remove the sample(s) from the weighted holder.

8.4.4 Place the sample(s) inside a glass beaker of soapy water and perform ultrasonic cleaning for 5 minutes.

8.4.5 Place the samples(s) inside a glass beaker of clean DIW and perform ultrasonic cleaning for 5 minutes.

8.4.6 Remove the sample(s) and dry with a clean, soft cloth.

\subsection{Final Polishing Bare U-Mo Samples using 50/50 Colloidal Silica and DIW with Vibratory Polisher}

8.5.1 Reload sample(s) into a clean weighted holder as directed in the instruction manual, using the sample-loading fixture.

8.5.2 Once loaded, place the weighted holder with sample(s) into the second vibratory polisher (50/50 colloidal silica and DIW), and carry out polishing for 24 hours.

8.5.3 When the polishing is complete, remove the sample(s) from the weighted holder.

8.5.4 Immediately clean the sample(s) by spraying with DIW and place it inside a clean glass beaker of DIW. Care must be taken to make sure that the samples do not dry after being removed from the colloidal silica solution. 
8.5.5 Perform ultrasonic cleaning for 2 minutes.

\subsection{Final Polishing Bare U-Mo Samples using 1\% Acetic Acid and DIW}

8.6.1 A glass plate will be used as a polishing base to help maintain sample flatness.

8.6.2 Set up a portion (cut piece) of a new Final B polishing cloth on a glass plate. Spray DIW on the cloth and clean off any loose fibers.

8.6.3 Apply $1 \%$ acetic acid and DIW mixture to the polishing cloth. Hold the sample by hand and manually polish for 1 minute.

8.6.4 Immediately clean the sample(s) with DIW and place inside a clean glass beaker of ethanol. Perform ultrasonic cleaning for 2 minutes.

8.6.5 Remove the bare U-Mo sample(s) and dry with canned air or warm air to prevent water spots on the surface. Store the prepared sample(s) properly using a properly labeled container.

\subsection{Cleanup after Final Polishing Bare U-Mo Samples}

8.7.1 After completing the final polishing process, clean the glass plate, specimen holders and loading plate/fixture.

8.7.2 If polishing media inside the vibratory polishers are not to be reused, absorb them using terry towels and place the used towels in an appropriate container (i.e., double plastic bag). Gently remove the polishing cloths from the vibratory polishers. Wipe the inner surface of the vibratory polishers to remove any oils or contamination.

8.7.3 Remove the absorbent material covering the work area and place it in the waste receptacle.

8.7.4 Wipe the exterior surface of the equipment and the surrounding work area with a damp towel.

8.7.5 Place the waste material in a waste container and document the addition of material on the waste tracking sheet for the container.

8.7.6 Arrange for a radiological survey. Based on the survey, determine whether the contamination is as low as reasonably achievable or further cleaning is required. 



\subsection{Sample Final Polishing - Plated U-Mo}

The purpose of final polishing on plated U-Mo mounted samples is to remove the surface damage only. It should not be used to remove any damage during sectioning and grinding operations. Plated U-Mo samples should be prepared using non-water-based lubricants and polishing suspensions.

\subsection{Equipment}

- Buehler VibroMet 2 vibratory polisher

\subsection{Supplies}

9.2.1 Polishing cloths

- Buehler nylon 12", PSA backing (\# 40-7072)

- Wendt Dunnington (3M) 12" Final B adhesive backing (\# 150237)

- Buehler ChemoMet 8" polishing cloth, PSA backing (\# 40-7918)

9.2.2 Polishing solutions/paste

- Buehler MetaDi II monocrystalline-diamond medium-concentration paste, 1 micron (\# 40-6243)

- Wendt Dunnington (3M) lapping oil OS114 (\# 185029)

9.2.3 Others

- Buehler weighted specimen holder 1.25" (\# 67-1527)

- Buehler loading plate/fixture (\# 67-1540)

- ultrasonic sample cleaner (example: Branson Model 2800)

- soft cloth

- terry towels

- glass plate

- ethanol

- hexane

- glass beaker

\subsection{Final Polishing Plated U-Mo Samples using 1 Micron Vibratory Polisher}

9.3.1 Prepare the vibratory polisher by properly applying nylon cloth without any wrinkles. Care must be taken to ensure a flat surface without any air bubbles.

9.3.2 Apply 1-micron diamond paste onto the cloth and then fill the basin as appropriate with the lapping oil. 
9.3.3 Turn on the vibratory polisher and let it run for 15 minutes before inserting sample(s).

9.3.4 Load the weighted holder as directed using the sample loading plate.

9.3.5 Once loaded, place the weighted holder with sample(s) into the first vibratory polisher ( 1 micron) and carry out polishing for 24 hours.

9.3.6 When the polishing (1 micron) is complete, remove the sample(s) from the weighted holder.

9.3.7 Place the sample(s) inside a glass beaker of hexane and perform ultrasonic cleaning for 3 minutes.

9.3.8 Clean the sample(s) using ethanol and dry with compressed air.

9.3.9 Place the sample(s) inside a glass beaker of ethanol and perform ultrasonic cleaning for 3 minutes.

9.3.10 Remove and clean the sample(s) with ethanol, dry with compressed air and wipe with a clean, soft cloth.

\subsection{Cleanup after Final Polishing}

9.4.1 After completing the final polishing process, clean the glass plate, specimen holders and loading plate/fixture.

9.4.2 If polishing media inside the vibratory polisher is not to be reused, absorb them using terry towels and place the towels in an appropriate container (i.e., double plastic bag). Gently remove the polishing cloths from the vibratory polisher. Wipe the inner surface of the vibratory polisher to remove any oils or contamination.

9.4.3 Remove the absorbent material covering the work area and place it in the waste receptacle.

9.4.4 Wipe the exterior surface of the equipment and the surrounding work area with a damp towel.

9.4.5 Place the waste material in a waste container and document the addition of material on waste tracking sheet for the container.

9.4.6 Arrange for a radiological survey. Based on the survey, determine whether the contamination is as low as reasonably achievable or further cleaning is required. 


\subsection{Optical Microscope Operating Procedure}

Optical metallography is the study of metals using an optical microscope. Structures that are coarse enough to be discernible to the naked eye or under low magnifications are termed macrostructures, whereas those that require high magnification to be visible are termed microstructures. Microscopes are required for the examination of a metal's or alloy's microstructure.

\subsection{Equipment}

- Olympus BX61M optical microscope or similar unit

- three-axis automated stage is preferred

- objectives: $2.5 \times, 5 \times, 10 \times, 20 \times$, and $50 \times$

- polarizing and differential interference contrast (DIC) filters

- digital camera, CCD (charge-coupled device) preferred

- Stream Motion software or other based on the microscope model

\subsection{Sample Condition}

Both bare U-Mo and plated U-Mo samples should be prepared according to the instructions mentioned earlier.

\subsection{Optical Metallography on Bare U-Mo Samples}

- In the case of bare U-Mo samples, once the final polishing is complete, store the samples for about 48 hours prior to examining them using an optical microscope. Typically, the grains show up much better in the polarized mode when these samples are oxidized in air for about 48 hours.

- Optical metallography will be performed in bright-field mode.

- A typical image series will include images at all five magnifications $(2.5 \times, 5 \times, 10 \times, 20 \times$, and $50 \times$ ) from three regions (left, center, and right) of the sample, as well as overview montages taken at $2.5 \times, 5 \times, 10 \times$, and/or $20 \times$.

- Good quality images can be obtained by using the polarized light filter.

- Refer to Section 12 (Atlas of Microstructures) to see typical microstructures in various conditions.

\subsection{Optical Metallography on Plated U-Mo Samples}

- Optical metallography will be performed in bright-field mode.

- A typical image series will include images at all five magnifications $(2.5 \times, 5 \times, 10 \times, 20 \times$, and $50 \times$ ) from three regions (left, center, and right) of the sample, as well as overview montages taken at $20 \times$.

- Good quality images can be obtained by using the polarized light filter. 
- Refer to Section 12 (Atlas of Microstructures) to see typical microstructures in various conditions. 


\subsection{Scanning Electron Microscope Operating Procedure}

\subsection{Instrumentation}

The following instruments (or similar) can be used to examine the U-Mo samples:

11.1.1 JEOL JSM-7600F with the following attachments

a. in-lens secondary detector (SEI)

b. Everhart-Thornley detector (lower electron image, LEI)

c. low-angle backscattered electron detector (LABE or LBE)

d. silicon-drift (SDD) energy-dispersive x-ray spectroscopy (EDS) detector

e. wavelength-dispersive $\mathrm{x}$-ray spectroscopy detector (WDS)

f. electron backscatter diffraction (EBSD) detector

11.1.2 Oxford Instruments AZtec system
a. $\quad \mathrm{X}-\operatorname{Max} 80 \mathrm{~mm}^{2} \mathrm{SDD}$ detector (item d. above).
b. Wave WDS detector (item e. above).
c. HKL Nordlys EBSD camera (item f. above).
d. AZtec software, version 2.2 SP1 or higher.

11.1.3 Structure Probe, Inc. (SPI) Module Sputter Coater

a. gold target

11.1.4 Fischione Model 1400 plasma cleaner

\subsection{General Conditions and Operating Parameters}

- Clean the polished U-Mo samples mounted in epoxy resin in a Fischione Plasma Cleaner for 5 minutes and then coat with $\sim 10 \mathrm{~nm}$ Au in an SPI-Module sputter coater. Clean the sample again for 5 minutes in the plasma cleaner.

- Attach a small piece of copper tape to the surface of the epoxy mount. This will be used for beam optimization of the EDS detector. Place the coated sample in a JEOL topreference holder and then insert into the vacuum chamber of the JEOL JSM-7600F scanning electron microscope.

- Place the sample at $15 \mathrm{~mm}$ working distance (WD) from the objective pole piece to collect the majority of data. 
- Set the microscope as follows: $20 \mathrm{keV}$ accelerating voltage, $110 \mu \mathrm{m}$ objective aperture, and a nominal beam current of $\sim 6 \times 10^{-9} \mathrm{~A}$, as listed in the JEOL microscope documentation.

- Some images, particularly those at higher magnifications, can be taken as follows: $8 \mathrm{~mm}$ $\mathrm{WD}, 30 \mu \mathrm{m}$ objective aperture, and a nominal beam current of $4.5 \times 10^{-11} \mathrm{~A}$.

- Compositional maps and data can be collected using the above analytical conditions, with a nominal dead time of $30-60 \%$.

- Once the beam current and acquisition settings are complete on the EDS software, perform a beam-current measurement (Beam Optimization in AZtec) using a piece of copper tape on the edge of the sample holder or on the epoxy mount.

- Collect images and maps using a resolution setting of 2048.

- Collect maps until there are approximately 50,000,000 to 100,000,000 counts in the Sum Spectrum. Collect point spectra for 60 seconds live time.

- Prepare a custom standard made from U-10Mo material and analyze it by using an external agency such as Southwest Research Institute. Store this data in the standards database of the AZtec system. This is a one-time operation that should be done prior to analysis. Recalibration should not be required, provided that no changes are made to the EDS detector hardware, but the standard may be used for Quality Assurance purposes.

- Collect montage images in Low Mag mode (LM) on the scanning electron microscope (SEM) and by using the "automate" feature of the AZtec software with a magnification setting of $100 \times$ or the lowest magnification that avoids clipping of the image by the LABE detector, whichever is higher. Collect a montage image at $250 \times$ in standard imaging mode at $15 \mathrm{~mm}$ WD.

- Collect the following stand-alone SEM images: $100 \times$ and $250 \times$ in LM; 500×, 800× and $5 \mathrm{k} \times$ in SEM mode. Other magnifications can be used as needed for observing specific features.

- LM in the JEOL JSM-7600F turns off the objective lens and uses the Angle Control lens for focus. It provides imaging from $25 \times$ to $10 \mathrm{k} \times$ with coarse focusing steps. SEM mode utilizes all of the lenses and provides much finer focusing capability and high-resolution imaging.

\subsection{Operating Procedures (Specific to JSM-7600F and Oxford Aztec 2.2)}

\subsubsection{Microscope Alignment (JSM-7600F specific)}

11.3.1.1 Insert the sample into the SEM as per SEM instructions.

11.3.1.2 Wait for vacuum to reach $4.4 \times 10^{-4} \mathrm{~Pa}$ or lower on the Penning gauge.

11.3.1.3 Set microscope conditions as follows: $20 \mathrm{keV}, 15 \mathrm{~mm} \mathrm{WD}, 110 \mu \mathrm{m}$ aperture, beam current of $\sim 6 \times 10^{-9} \mathrm{~A}$ or higher. Higher beam currents allow for more rapid EDS data acquisition. 
11.3.1.4 Turn on the beam and focus the image with Z-Axis control at a low magnification.

11.3.1.5 Align the source at Probe Current setting HC20 for the brightest image or highest current using the probe current detector, if available.

11.3.1.6 Insert the LABE detector.

11.3.1.7 Align the beam at Probe Current setting HC14-HC16 to remove the image shift during OL oscillation.

11.3.1.8 Align stigmator centers at Probe Current setting HC14-HC16 to remove the image shift during stigmator oscillation.

11.3.1.9 Set the magnification to $250 \times$ and center a feature.

11.3.1.10 Switch to the LM and set magnification to $250 \times$.

11.3.1.11 Center a feature using the LM Center alignment.

11.3.1.12 Return to the SEM mode and recheck beam alignment and stigmator centers.

\subsubsection{Microscope Operation}

11.3.2.1 Locate center of the sample. Adjust the focus and stigmation at $15-20 \mathrm{k} \times$.

11.3.2.2 Acquire images at the desired magnification settings, shifting the sample as needed to stay clear of carbon tracks and polishing artifacts. On the JSM-7600F, in SEM mode, images are available from the SEI, LEI, and LABE detectors, whereas LM mode only offers LEI and LABE images.

\subsubsection{EDS Setup}

11.3.3.1 Record the stage location of the Region of Interest.

11.3.3.2 Move to a piece of copper tape and set to $15 \mathrm{~mm}$ WD by focusing with the Z-axis control.

11.3.3.3 Switch to the Optimization Menu on the AZtec software.

11.3.3.4 Select "Copper" in the Element drop-down menu and collect optimization spectrum. Save optimization.

\subsubsection{EDS Acquisition}

11.3.4.1 Return to the recorded stage position for the Region of Interest.

11.3.4.2 Set the microscope to the SEM mode and WD to $15 \mathrm{~mm}$ (or analytical WD for your system).

11.3.4.3 Focus the image using the Z-axis control. 
11.3.4.4 Set the EDS software to achieve a dead time of 30-60\% for an SDD detector and $25-30 \%$ for a $\mathrm{Si}(\mathrm{Li})$ detector.

11.3.4.5 Set the microscope to the desired magnification and acquire a reference image.

11.3.4.6 Collect maps for a total number of counts between 50,000,000 and $100,000,000$.

11.3.4.7 Collect point spectra for 60 seconds live time with a setting of 2048 channels. 


\subsection{Atlas of Microstructures}

Typical micrographs generated from the BX61M optical microscope taken without and with polarizing and DIC filters are shown in Figure 1 below. The difference in grains and features is evident with and without the DIC filter.

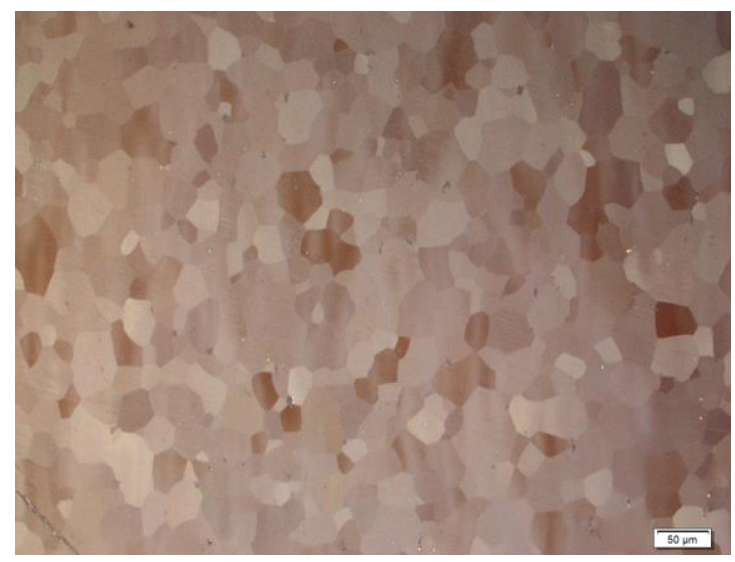

(a) Unfiltered Light

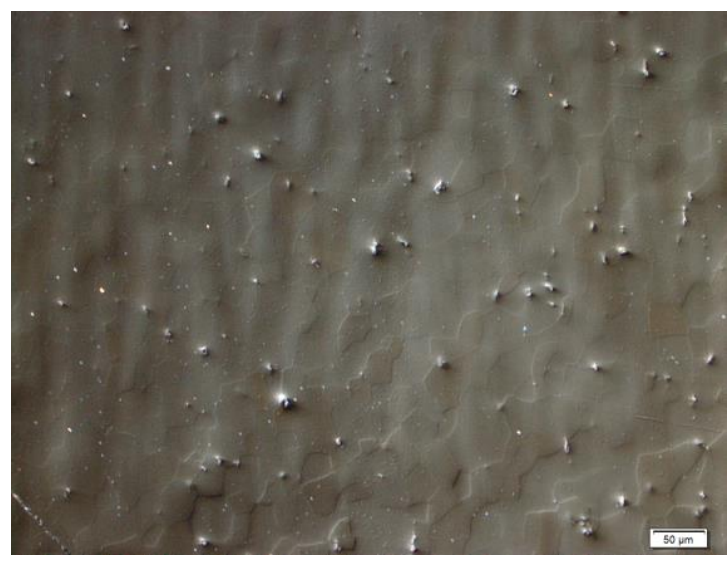

(b) Polarizing Light and DIC Filters

Figure 1. Optical Micrographs with and without Filters 


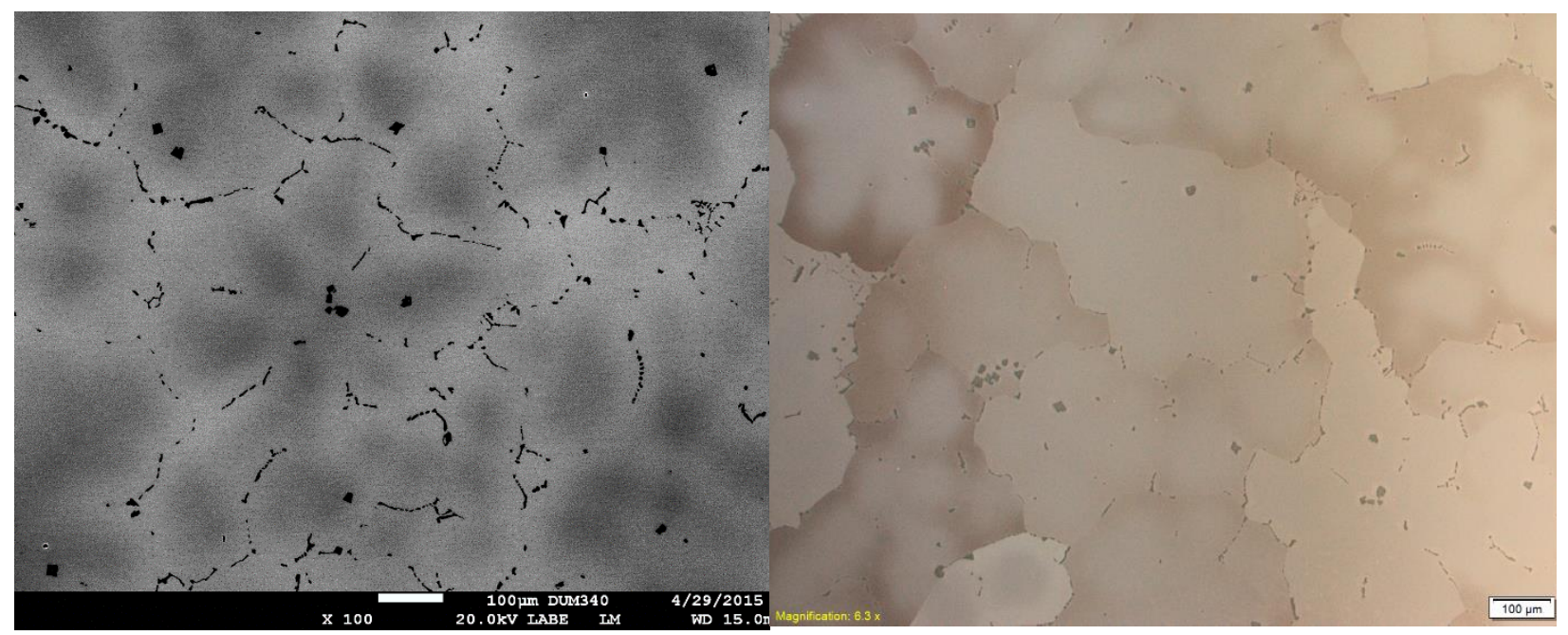

(a)

(b)

Figure 2. As-Cast Microstructure

The images above show an as-cast U-10Mo plate with a dendritic structure, which was cast at the Y-12 National Security Complex (Sample 3k32-X1-X21D).

Figure 2a is a backscattered electron (BSE)-SEM image while the image in Figure $2 \mathrm{~b}$ is the optical image taken under polarized light.

In Figure 2a, the bright regions are molybdenum-lean regions, whereas the gray regions are molybdenumrich regions. This is due the Z-contrast (atomic number contrast).

The reverse holds true for the optical images (Figure 2b), i.e., the molybdenum-lean regions are dark and molybdenum-rich regions are bright.

The addition of molybdenum improves the corrosion resistance, i.e., there is less oxidation, and it causes less dispersion of the light optically than is seen in the molybdenum-lean regions.

The black particles in both the images are uranium carbides (usually) and in some cases, oxides. 


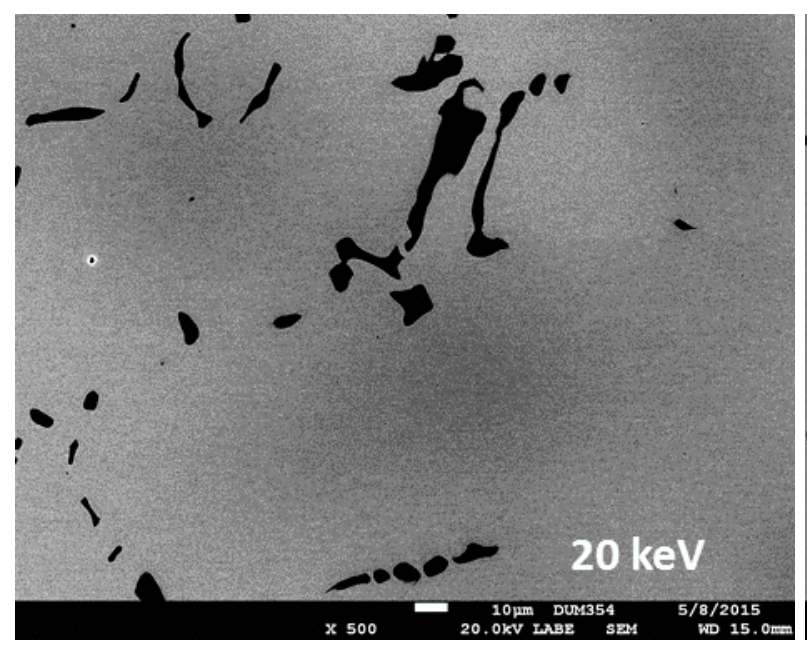

(a)

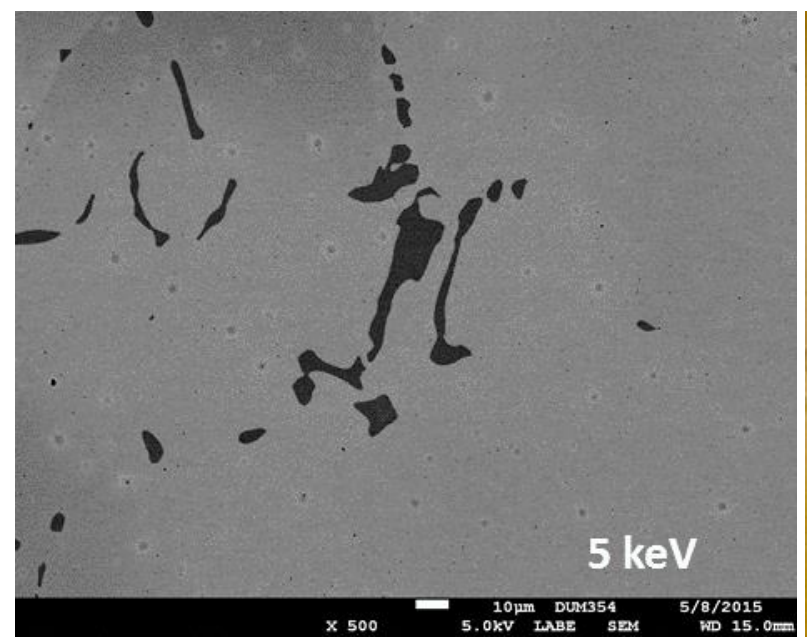

(c)

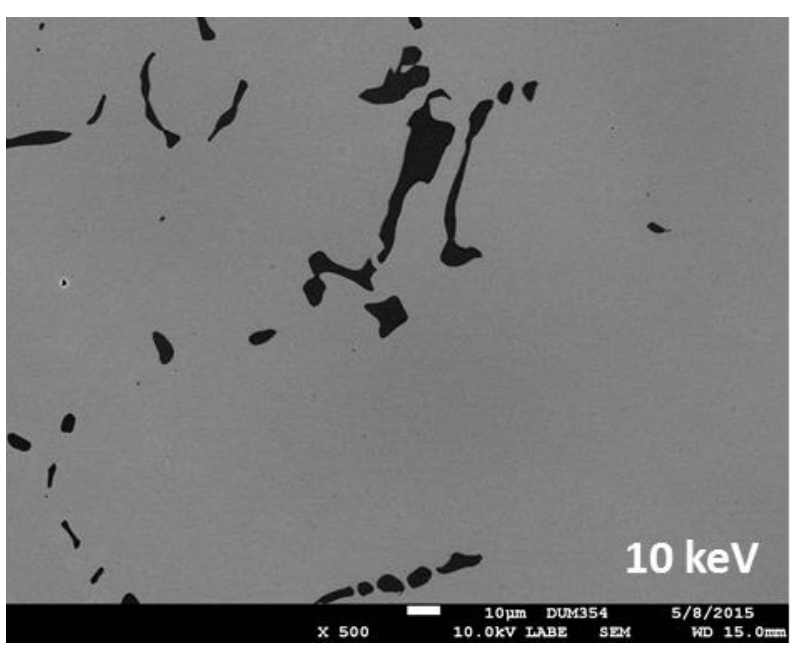

(b)

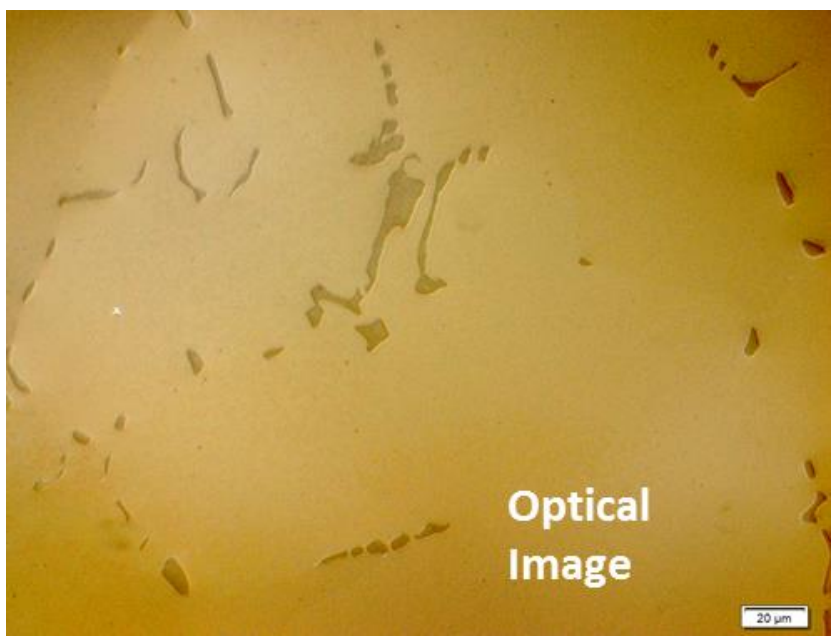

(d)

Figure 3. As-Cast Microstructure: Effect of Electron Voltage on Microstructure

Figure 3 shows the effect of electron beam potential on the microstructure (BSE-SEM images).

Due to the high $\mathrm{Z}$ of uranium, the interaction region of electrons is small.

Application of higher potential (more than $20 \mathrm{keV}$; Figure 3a) reveals the molybdenum inhomogeneity more prominently than with lower voltages (Figure $3 \mathrm{~b}$ and Figure $3 \mathrm{c}$ ).

Optically (Figure 3d), depending on the oxidation of the surface, the molybdenum inhomogeneity is revealed more prominently.

The carbides (black particles) are prominent and similar at all potentials of the SEM and under the optical microscope. 


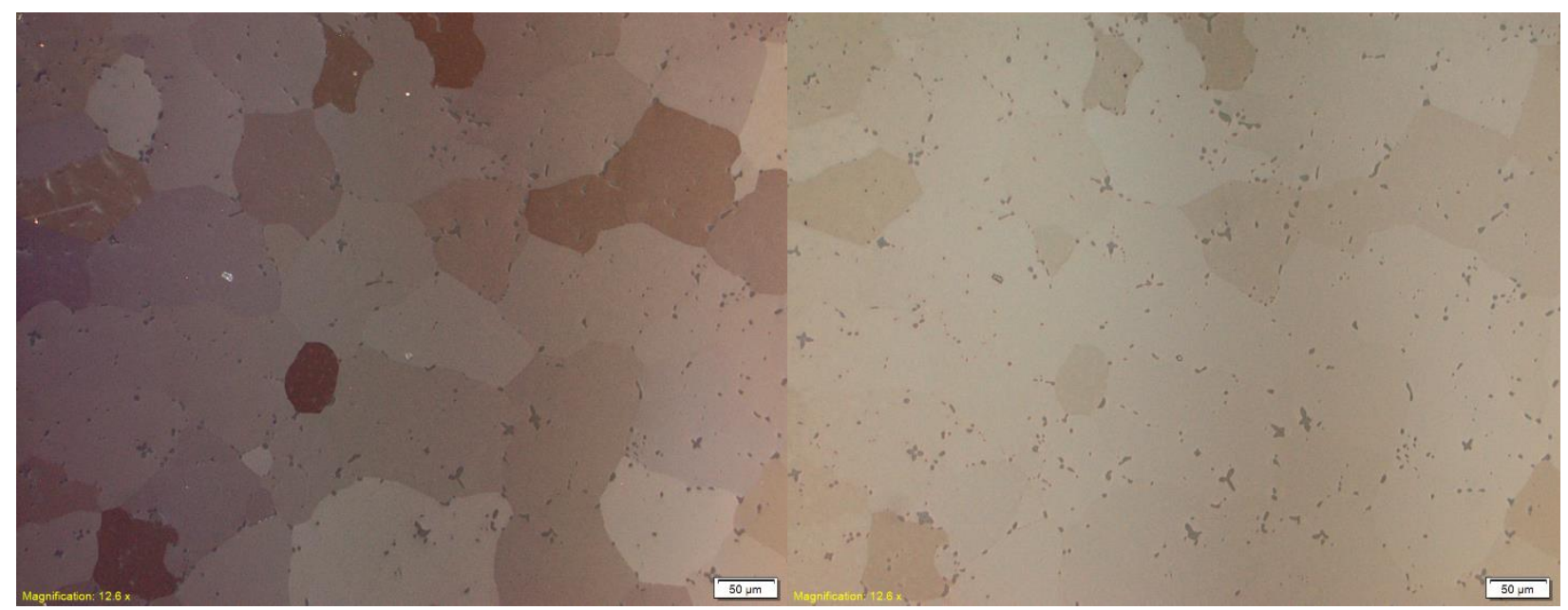

(a)

(b)

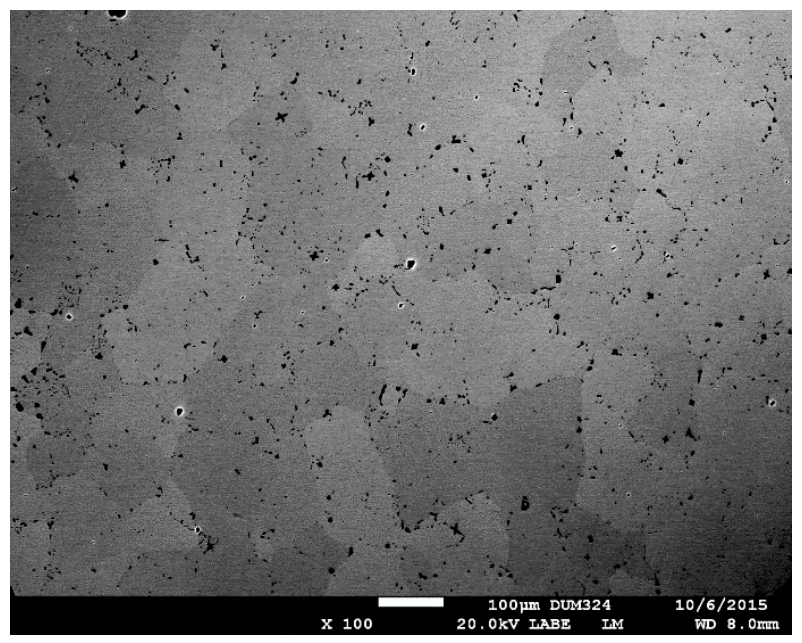

(c)

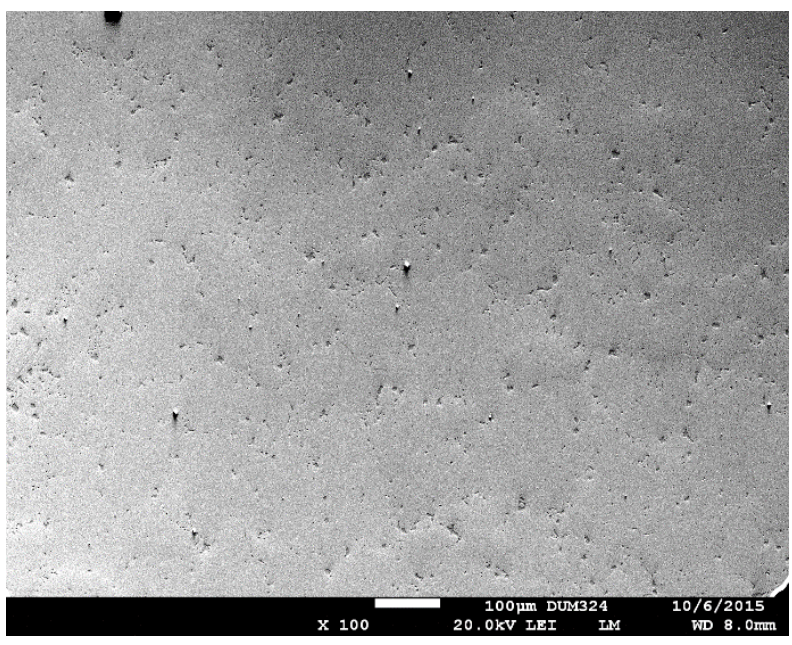

(d)

Figure 4. U-10Mo Microstructure: (a-b) Effect of Polarized Light; (c-d) BSE-SEM vs. Secondary Electron Images

Figure 4 shows a microstructure of U-10Mo plate (cast at Y-12 National Security Complex - sample Andrew 3rd). The sample was annealed at $1000^{\circ} \mathrm{C}$ for $4 \mathrm{~h}$.

Figure $4 \mathrm{a}$, on the top left, is an optical image with the polarizing filter, whereas Figure $4 \mathrm{~b}$, on the top right, is without the polarizing filter. The grains are distinctly visible in the polarized image as compared to the grains in the image where the polarizing filter was not used.

The BSE-SEM image (Figure 4c) does provide some information about the grain size, but is very difficult to discern; however, it shows Mo inhomogeneity more clearly (dark and light gray regions).

Figure $4 \mathrm{~d}$ is the secondary electron image of the region shown in Figure 4c; it shows carbides and the quality of the samples prominently. 


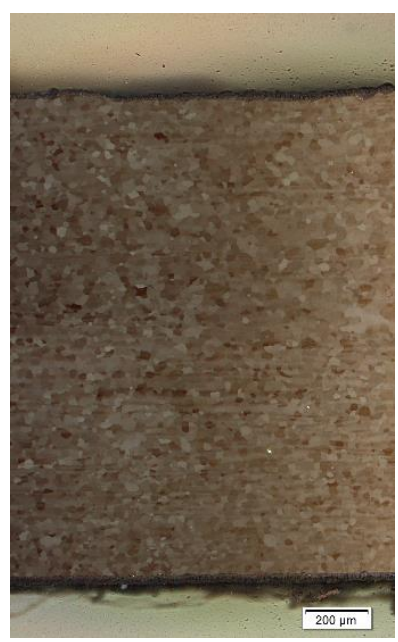

(a)

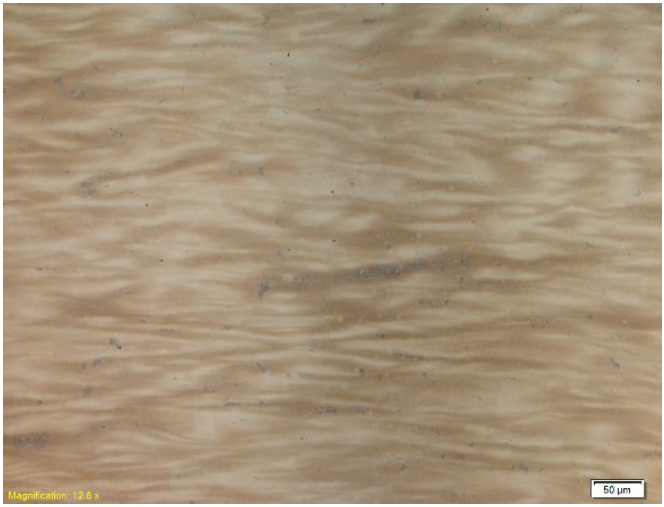

(c)

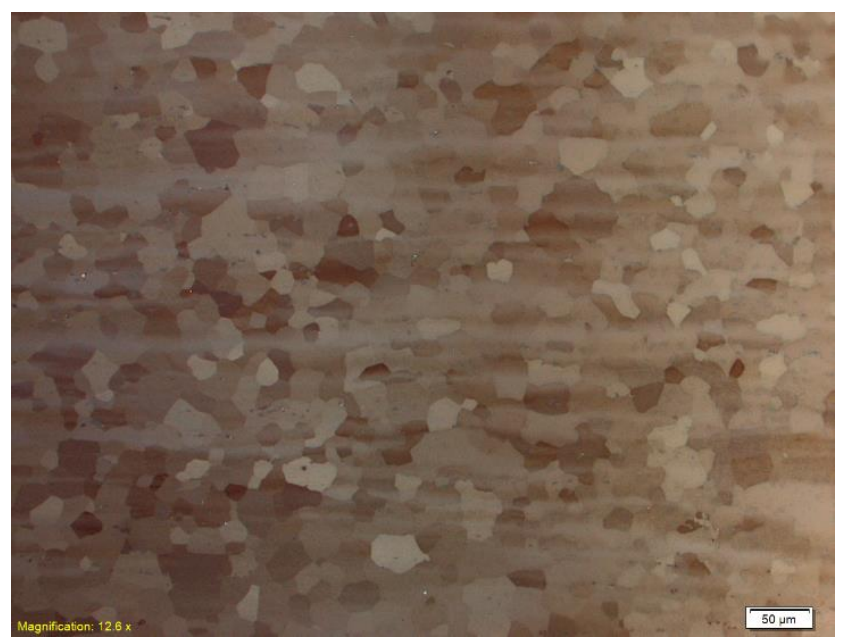

(b)

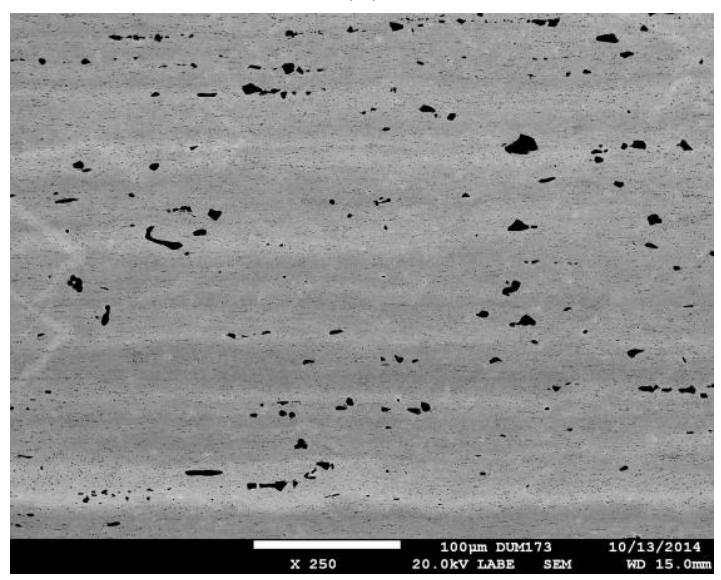

(d)

Figure 5. U-10Mo Microstructure: As-Cast and Rolled Microstructure

Figure 5 shows the microstructure of the as-cast and hot-rolled sample.

Figure 5a and Figure 5b are the optical images under polarized light, whereas Figure 5c is under nonpolarized light. Figure $5 \mathrm{~d}$ is a BSE-SEM image.

The polarized images are good for revealing the grains after the samples are oxidized in air for $48 \mathrm{~h}$.

The flow lines generated due to inhomogeneity are visible under the polarized light.

The molybdenum inhomogeneity can be observed in Figure 5c and Figure 5d, though the grains are not necessarily visible. 


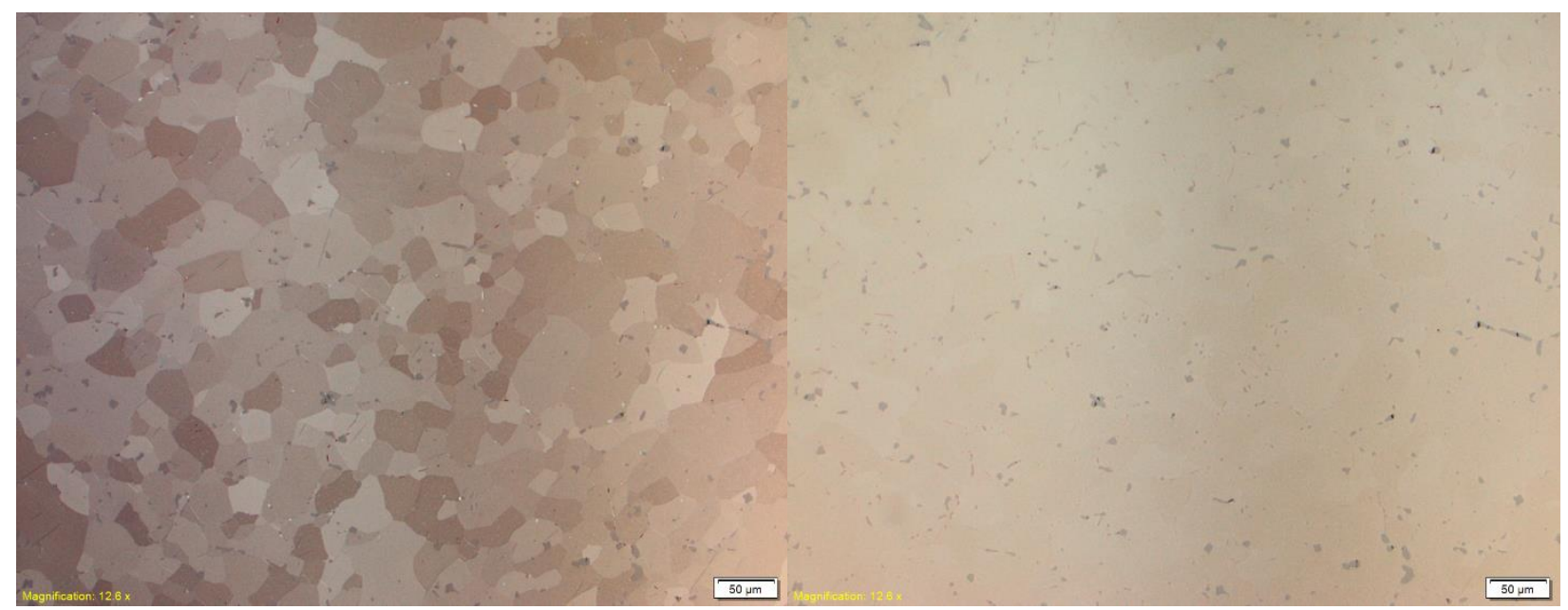

(a)

(b)

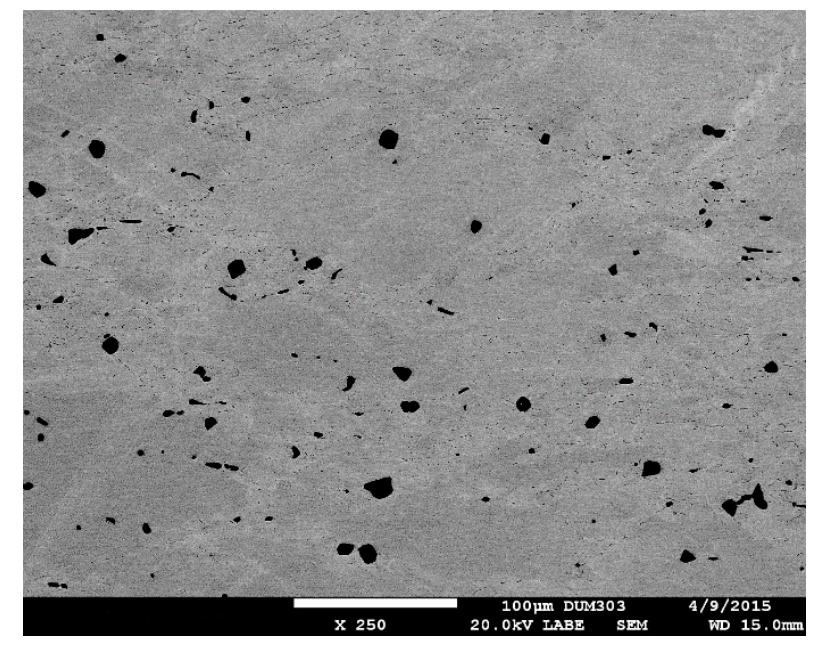

(c)

Figure 6. U-10Mo Microstructure: As-Cast, Homogenized, and Rolled Microstructure

The images in Figure 6 show the microstructure of the homogenized, as-cast and hot-rolled sample.

Figure $6 \mathrm{a}$ is the optical image under polarized light, whereas Figure $6 \mathrm{~b}$ is under non-polarized light. Figure $6 c$ is a BSE-SEM image.

The polarized images are good for revealing the grains after the samples are oxidized in air for $48 \mathrm{~h}$.

The molybdenum homogeneity does not create any banded structure and presents a clean microstructure under the BSE-SEM imaging. 


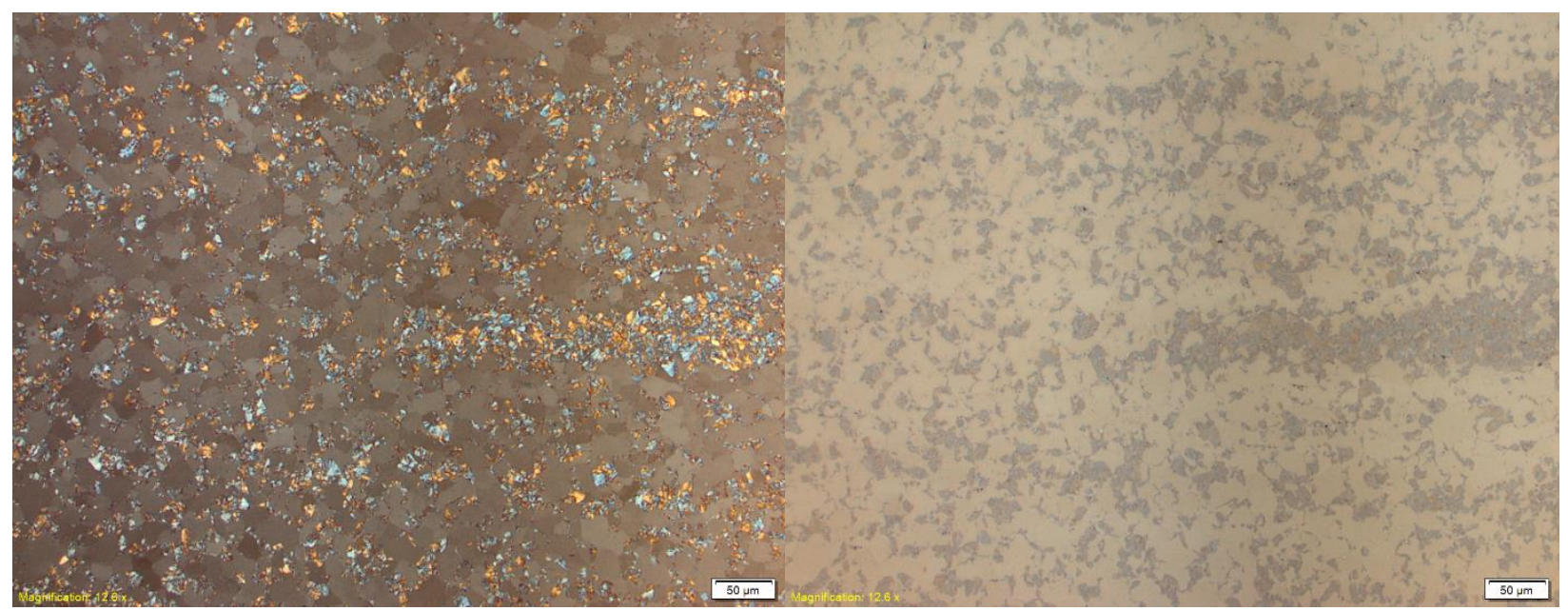

(a)

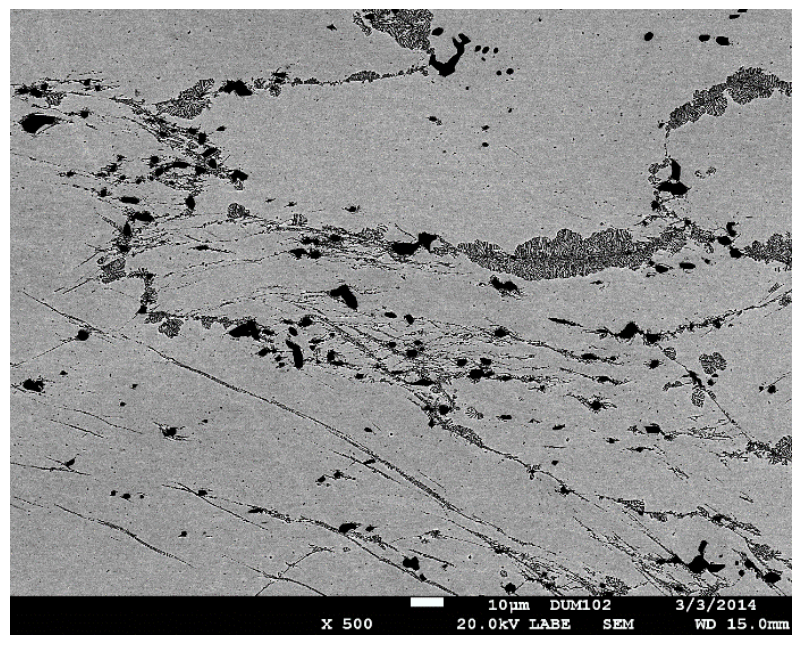

(c)

(b)

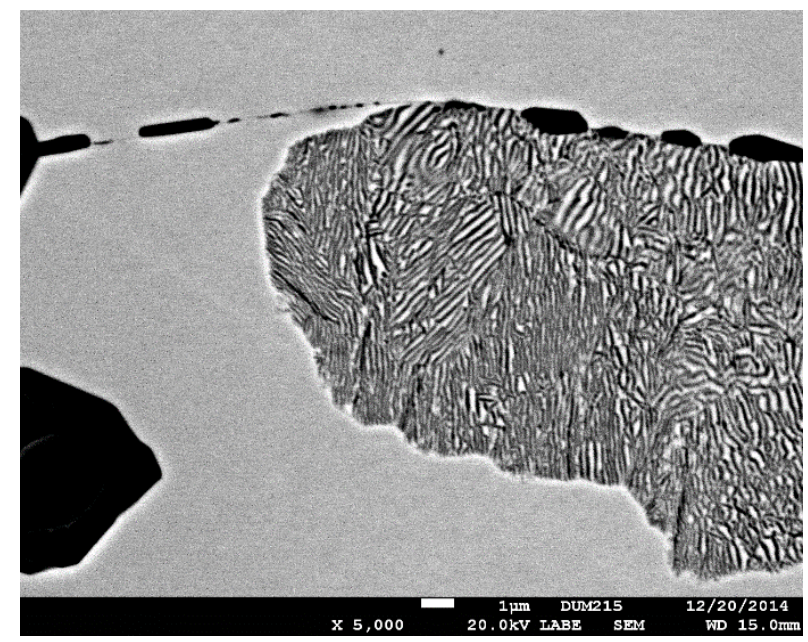

(d)

Figure 7. U-10Mo Microstructure: Eutectoid Structure

The images in Figure 7 show the microstructure of the eutectoid structure.

Figure $7 \mathrm{a}$ is the optical image under polarized light whereas Figure $7 \mathrm{~b}$ is under non-polarized light.

Figure 7c and Figure 7d are high- and low-magnification BSE-SEM images, respectively. 


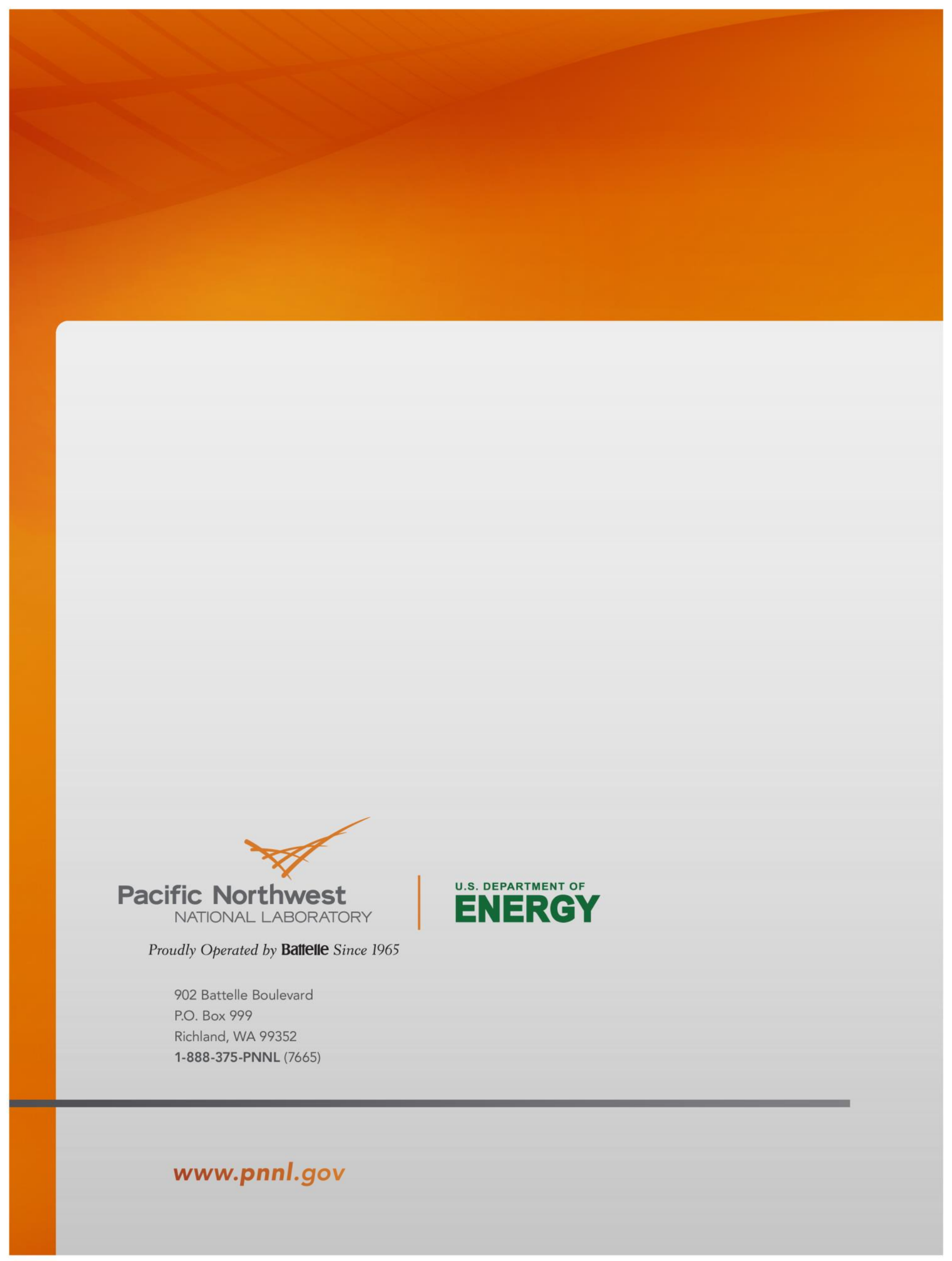

